\title{
Fluid Mechanics and Optics: the effect of turbulence in the observation of the Universe
}

\author{
Dr. Ramon Codina \\ Polytechnic University of Catalonia, Spain
}

In this talk we describe the importance of aerodynamics in the optimal properties of the atmosphere and their implications in the selection of the location of large astronomic facilities. We present a description of turbulence from the point of view of computational fluid dynamics, linking its modeling to the numerical formulation used to approximate the Navier-Stokes equations. We also explain how pressure, velocity and temperature fluctuations are used to compute the parameters that describe the quality of the seeing of a region, with a direct implication in the selection of telescope locations. 